\title{
Synchronization of Spatiotemporal Chaos Using Nonlinear Feedback Functions
}

\author{
M.K. ALI ${ }^{\mathrm{a}, *}$ and JIN-QING FANG ${ }^{\mathrm{b}}$ \\ ${ }^{a}$ Department of Physics, The University of Lethbridge, Lethbridge, Alberta TIK 3M4, Canada; ${ }^{\mathrm{b}}$ China Institute of Atomic Energy, \\ P. O. Box 275-27, Beijing 102413, P.R. China
}

(Received 20 February 1997)

\begin{abstract}
Synchronization of spatiotemporal chaos is studied using the method of variable feedback with coupled map lattices as model systems. A variety of feedback functions are introduced and the diversity in their choices for synchronizing any given system is exemplified. Synchronization in the presence of noise and with sporadic feedback is also presented.

Keywords: Chaos, Control, Synchronization, Feedback, Nonlinear, Spatiotemporal, Random
\end{abstract}

PACS Numbers: $05.45+\mathrm{b}$

Recently, there has been considerable progress [1] in controlling and synchronizing chaos in low dimensional systems. However, our current understanding of such issues in spatially extended systems with many competing degrees of freedom is very rudimentary. Spatially extended dissipative systems can manifest, under suitable conditions, behaviors that are (1) predictable, (2) chaotic in spatial and temporal (spatiotemporal) dimensions, or (3) fully random (turbulent). Spatiotemporal chaos appears in such diverse phenomena as plasma fusion, hydrodynamics, optics, chemical reactions, storage of memory in the brain, pattern formations and Raleigh-Bènard convection. For theoretical investigations, spatially extended systems are often modeled by partial differential equations, coupled ordinary differential equations, coupled map lattices (CMLs) and cellular automata. Of all these models, the CMLs are the simplest and have been found valuable in revealing many important features of spatiotemporal chaos. We will use the CMLs for our models here. Because of great potentials for practical applications, this area of research has been very active and several methods have been developed in this direction. For example, we have learnt about the OGY strategy [2], the Pecora-Carroll scheme with variable feedback

\footnotetext{
* Corresponding author.
} 
[3], small perturbation feedback control [4], the pinning feedback [5], the video-feedback control [6], the resonant technique [7], the modified Pecora-Carroll scheme with discrete time coupling [8]. These approaches have their strengths and weaknesses. For example, the pinning approach [5] has the limitation that it is effective only for certain ranges of values of the coupling strength $\epsilon$ and it has the undesirable feature that it gives rise to ambiguous expressions for the response state vectors when the pinning is applied to every or every other site. Although sporadic feedback may be desirable in some situations, we need to have methods to deal with cases where the feedback is also applied to every site or every other site. In this work, we present nonlinear feedback functions for model CMLs that work well and do not suffer from such limitation or ambiguity. The CML can be defined as

$$
\begin{gathered}
X_{n+1}(i)=\beta_{i} F\left(X_{n}(i)\right)+\frac{1}{\mu} \sum_{j=1, j \neq i}^{\mu} \epsilon_{i j} h\left(X_{n}\left(m_{i j}\right)\right), \\
i=1,2, \ldots, L, \quad \mu \leq L-1,
\end{gathered}
$$

where $F\left(X_{n}(i)\right)$ describes the internal dynamics of the $i$ th site when couplings with other sites are absent. In this work, $F(X)=4 X(1-X)$ is the one-dimensional logistic map. In Eq. (1), $i$ and $n$ denote discrete space and time variables and $L$ is the total number of sites each of which is connected to $\mu$ neighbors, $\beta_{i}$ is the $i$ th system parameter, $m_{i j}$ is the index of the $j$ th neighbor of the $i$ th site, $\epsilon_{i j}$ is the coupling (connection) strength of the $j$ th neighbor of the $i$ th site and $h\left(X_{n}\left(m_{i j}\right)\right)$ is a function of the state variables $X_{n}\left(m_{i j}\right)$. The dynamical behavior of a CML depends on the parameters $\beta_{i}$, the nature of coupling (type of $h$ and values of $m_{i j}$, the coupling strengths $\epsilon_{i j}$ and boundary conditions. We present results for (i) lattices with random neighbors and fixed coupling strength (CMLR) and (ii) lattices with random neighbors and random coupling strengths (CMLRR). We have also studied the lattices of $[5,9,10]$ and have found that our scheme of gen- erating feedback functions (see below) work equally well for these lattices. For the CMLR and CMLRR, the $m_{i j}$ were found by a random number generator and we set $h\left(X_{n}\left(m_{i j}\right)\right)=F\left(X_{n}\left(m_{i j}\right)\right)$ with $\beta_{i}=1-\epsilon, i=1,2, \ldots, L$. The goal here is to synchronize the driver system of Eq. (1) with the response system

$$
\begin{aligned}
Y_{n+1}(i)= & \beta_{i} F\left(Y_{n}(i)\right) \\
& +\frac{1}{\mu} \sum_{j=1, j \neq i}^{\mu} \epsilon_{i j} h\left(Y_{n}\left(m_{i j}\right)\right)+G_{n}(i),
\end{aligned}
$$

where $G_{n}(i)$ is the feedback function at the $i$ th site. Starting from different random initial values of the state vectors $\mathbf{X}_{0}$ and $\mathbf{Y}_{0}\left(\mathbf{X}_{0} \neq \mathbf{Y}_{0}\right)$, one iterates Eqs. (1) and (2) simultaneously. When synchronization between the driver and response systems is achieved, $\mathbf{X}_{\mathrm{n}}=\mathbf{Y}_{\mathrm{n}}$ and the feedback function $G_{n}(i)=0$. The feedback functions are not unique. Different feedback functions that synchronize a given CML are obtained by replacing [11] $X_{n}(i)\left(1-X_{n}(i)\right)$ in Eq. (1) with suitable functions $g_{n}(i)$. Some examples of suitable $g_{n}(i)$ are given below:

$$
\begin{aligned}
& g_{n}(i)=\left(Y_{n}(i)-X_{n}(i)\right)\left(2 X_{n}(i)-1\right), \\
& g_{n}(i)=\frac{1}{2}\left(Y_{n}(i)-X_{n}(i)\right)\left(3 X_{n}(i)^{2}-1\right), \\
& g_{n}(i)=\frac{1}{2}\left(Y_{n}(i)-X_{n}(i)\right)\left(4 X_{n}(i)^{2}-1\right), \\
& g_{n}(i)=\tanh \left[\left(Y_{n}(i)-X_{n}(i)\right)\left(2 X_{n}(i)-1\right)\right], \\
& g_{n}(i)=\left(Y_{n}(i)-X_{n}(i)\right) \tanh \left[\left(2 X_{n}(i)-1\right)\right], \\
& g_{n}(i)=\left(2 X_{n}(i)-1\right) * \tanh \left(Y_{n}(i)-X_{n}(i)\right), \\
& g_{n}(i)=\left(2 X_{n}(i)-1\right) * \sin \left(Y_{n}(i)-X_{n}(i)\right), \\
& g_{n}(i)=\sin \left[\left(Y_{n}(i)-X_{n}(i)\right)\left(2 X_{n}(i)-1\right)\right], \\
& g_{n}(i)=\left(Y_{n}(i)-X_{n}(i)\right) \sin \left[\left(2 X_{n}(i)-1\right)\right], \\
& g_{n}(i)=\left(Y_{n}(i)-X_{n}(i)\right)\left(2 X_{n}(i)-1\right)^{3},
\end{aligned}
$$

etc.

For a given $g_{n}(i)$, the feedback functions for the CMLR and CMLRR are, respectively, given by

$$
G_{n}(i)=\gamma\left[(1-\epsilon) g_{n}(i)+\frac{\epsilon}{\mu} \sum_{j=1, j \neq i}^{\mu} g_{n}\left(m_{i j}\right)\right]
$$


and

$$
G_{n}(i)=\gamma\left[(1-\epsilon) g_{n}(i)+\frac{1}{\mu} \sum_{j=1, j \neq i}^{\mu} \alpha_{i j} g_{n}\left(m_{i j}\right)\right]
$$

where $\gamma$ is an adjustable parameter. The $\alpha_{i j}$ in Eq. (14) for the CMLRR are random numbers with $0 \leq \alpha_{i j} \leq \epsilon$ and the symmetry condition $\alpha_{i j}=\alpha_{j i}$ is not invoked. During the iterations if $Y_{n+1}(i)$ falls outside the basin of attraction (here the interval $(0,1))$, then the contribution of the feedback term is scaled so that $Y_{n+1}(i)$ belongs to this interval or simply set $Y_{n+1}(i)=\left|Y_{n+1}(i)\right|$ modulo 1 . There is a trivial feedback function that reduces Eq. (2) to Eq. (1) identically. This happens when

$$
\begin{aligned}
G_{n}(i)= & \beta_{i} F\left(X_{n}(i)\right)+\frac{1}{\mu} \sum_{j=1, j \neq i}^{\mu} \epsilon_{i j} h\left(X_{n}\left(m_{i j}\right)\right) \\
& -\beta_{i} F\left(Y_{n}(i)\right)-\frac{1}{\mu} \sum_{j=1, j \neq i}^{\mu} \epsilon_{i j} h\left(Y_{n}\left(m_{i j}\right)\right) .
\end{aligned}
$$

For the logistic map this corresponds to

$$
\begin{aligned}
g_{n}(i)= & \left(Y_{n}(i)-X_{n}(i)\right)\left(\left(2 X_{n}(i)-1\right)\right. \\
& \left.+\rho\left(Y_{n}(i)-X_{n}(i)\right)\right)
\end{aligned}
$$

with $\rho=1$. Away from this trivial case, there is an infinite set of values of $\rho$ in the interval $\rho_{\min } \leq \rho \leq \rho_{\max }$ that will synchronize our CMLs

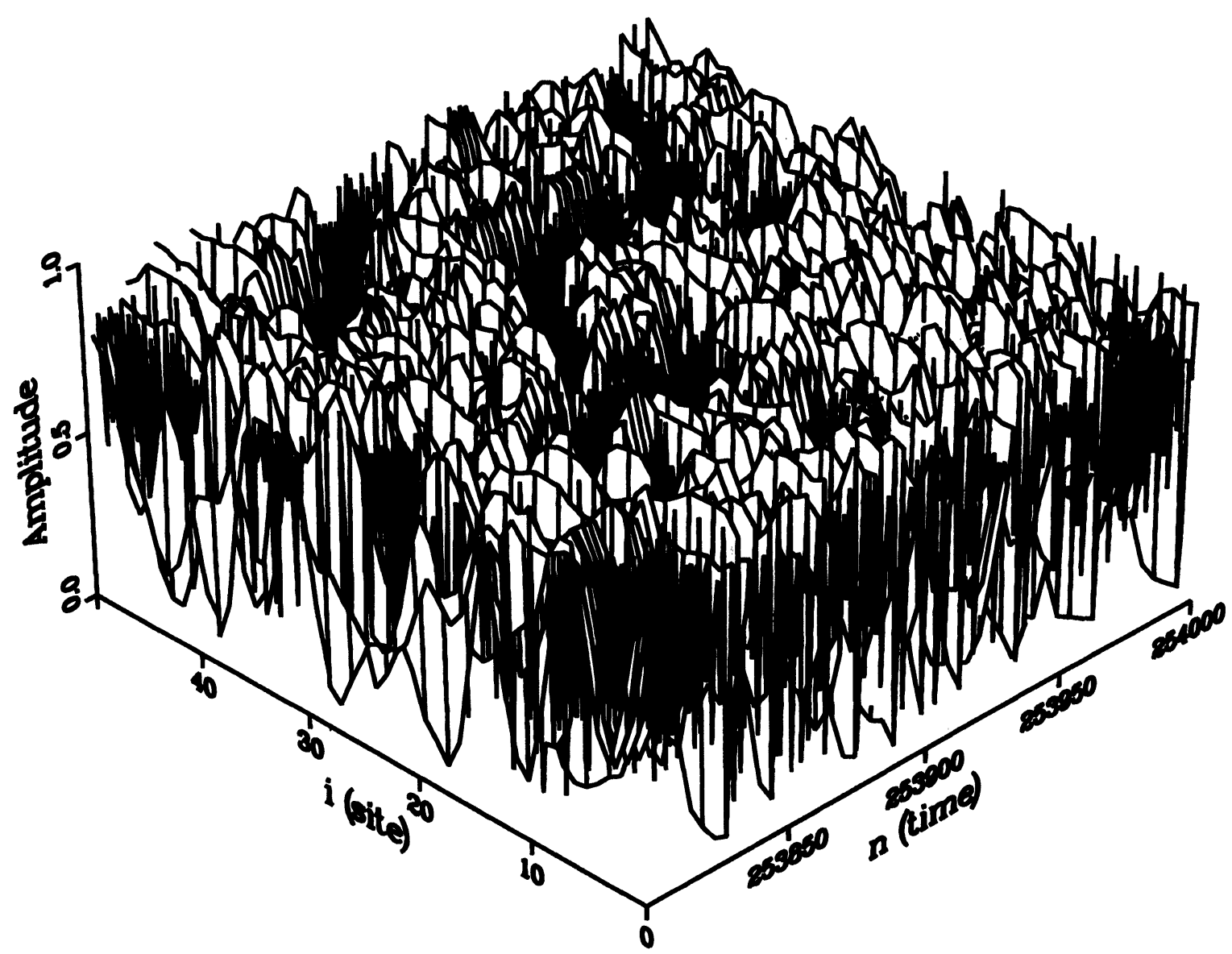

FIGURE 1 A typical spatiotemporal chaos for a CMLR as a function of discrete space and time variables $i$ and $n$. Here $L=50, N=20$ and $\epsilon=0.1$. It can be synchronized by using any of the $g_{n}(i)$ of in Eq. (13). 
with varied synchronization efficiencies. As for $\rho_{\text {min }}$ and $\rho_{\max }$ for the CMLRR with $L=50, \nu=10$, we could synchronize with $-1.2<\rho<2.8$. The feedback functions are applied to every site and at every iteration. For all the cases mentioned above, our feedback functions achieve synchronization for all values of $\epsilon$ in the interval $0<\epsilon<1$ and not only for certain ranges of values as in [5]. The number of neighbors $\mu$ in Eqs. (13) and (14) varied from 1 to $L-1$. When $L$ is large and $\mu$ is small, there is a chance to have disjoint groups of sites for random $m_{i j}$. To avoid this, we chose the first neighbor as the next neighbor and the remaining $\mu-1$ neighbors were chosen at random. Figure 1 shows a typical spatiotemporal chaos in a CMLR with $L=50, \mu=2$ and $\epsilon=0.1$. The feedback functions with any $g_{n}(i)$ from Eqs. (3)-(12) are found to synchronize spatiotemporal chaos in all the CMLs mentioned above. The rate of synchro- nization with different $g_{n}(i)$ are somewhat different. The transition time for a given CML is measured here by the number of iterations required for the function

$$
\Delta(n)=\sqrt{\sum_{i=1}^{L}\left(Y_{n}(i)-X_{n}(i)\right)^{2}}
$$

to reduce to a preset small number. For the CMLRR with $L=50$ and $\nu=10$, synchronization to the precision $\Delta(n) \leq 1 \times 10^{-10}$ required 19,18 , $51,15,18,18,20,12,20,268$ iterations for the feedback functions obtained from Eqs. (3)-(12) respectively. Figure 2 shows the transition times for sample feedback functions for the CMLRR. From Fig. 2, it can be seen that among the five feedback functions the one corresponding to Eq. (10) is the fastest while the one corresponding

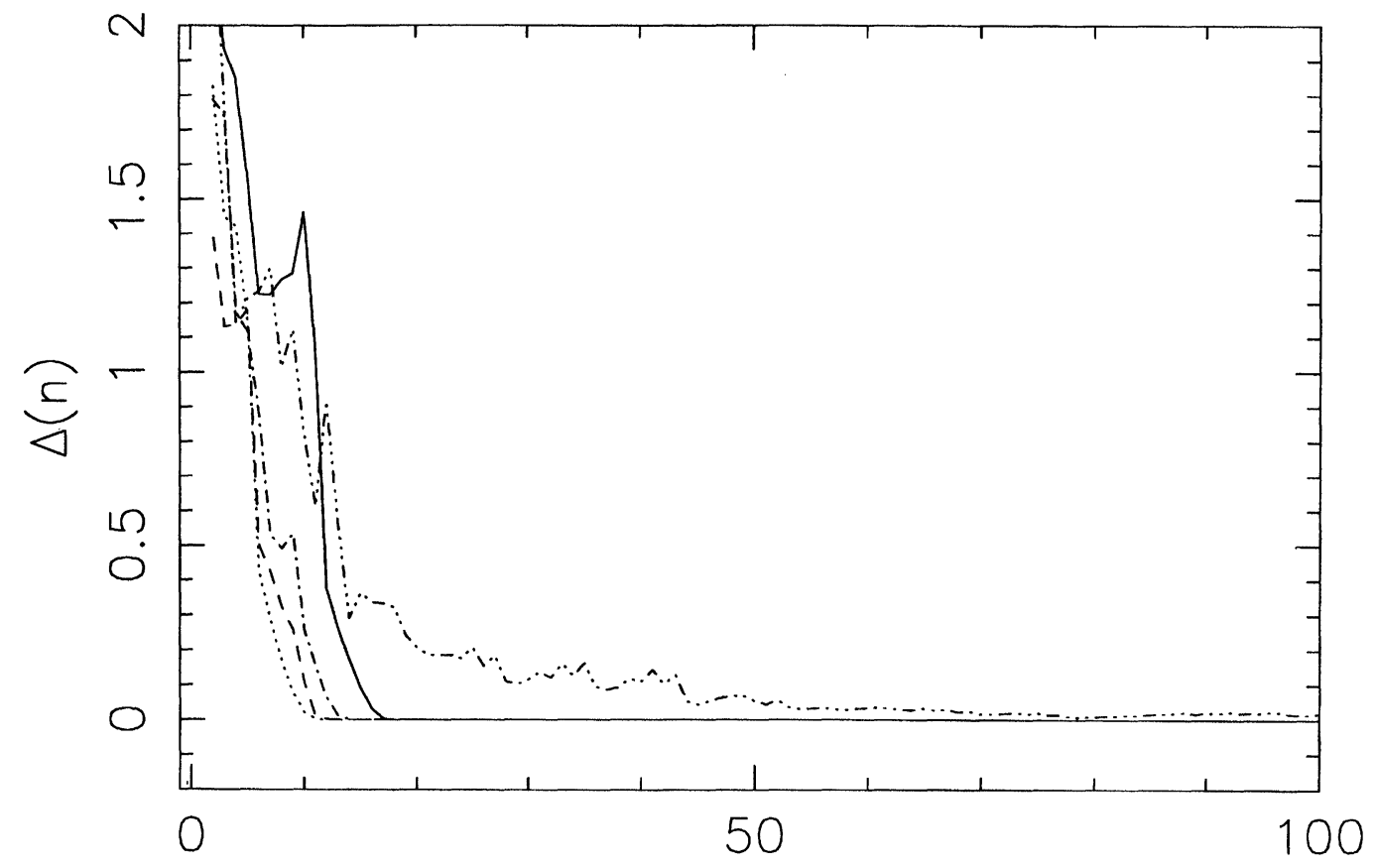

n

FIGURE 2 Transition time for sample feedback functions for CMLRR with $L=50, \nu=10, \gamma=4, \epsilon=0.1,0 \leq \alpha_{i j} \leq \epsilon$. The lines with styles - , - -.-- $\cdots$ and $-\cdots$ respectively, represent the feedback functions corresponding to Eqs. (4), (5), (6), (10) and (12). 
to Eq. (12) is the slowest. Results with other values of $L$ and $\mu$ for the CMLRR show similar trends in transition times.

So far we have considered synchronization of the CMLs with feedback applied to every site. This approach successfully synchronizes the CMLs for all values of $\epsilon$ in the interval $(0,1)$. For certain ranges of values of $\epsilon, \gamma$ and $L$, it is not necessary to apply the feedback at every site for realizing synchronization. To illustrate, we consider the lattice used in [5] for which our feedback function becomes

$G_{n}(i)=\gamma[(1-\epsilon)) f_{n}(i)+\frac{\epsilon}{2 N} \sum_{j=1}^{N}\left(f_{n}(i+j)+f_{n}(i-j)\right]$.
This CML, with $L=50, N=2, \gamma=2.2, \epsilon=0.8$ and periodic boundary conditions, can be synchronized by applying the feedback only at the 35 th site. This CML could also be synchronized by using any one of the (site, $\gamma$ ) pairs $(25,2.4)$, $(19,2.5),(14,2.5),(13,1.2),(10,2.5)$, and $(4,0.5)$. The same lattice with $N=1$ could be synchronized by adding feedback at an interval of two sites with $0.4<\epsilon \leq 1$. The success of sporadic feedback depends on the use of appropriate values of the system parameters.

Synchronization of spatiotemporal chaos in the presence of noise is of importance from experimental considerations. Our numerical results show that quasi-synchronization is realized in the

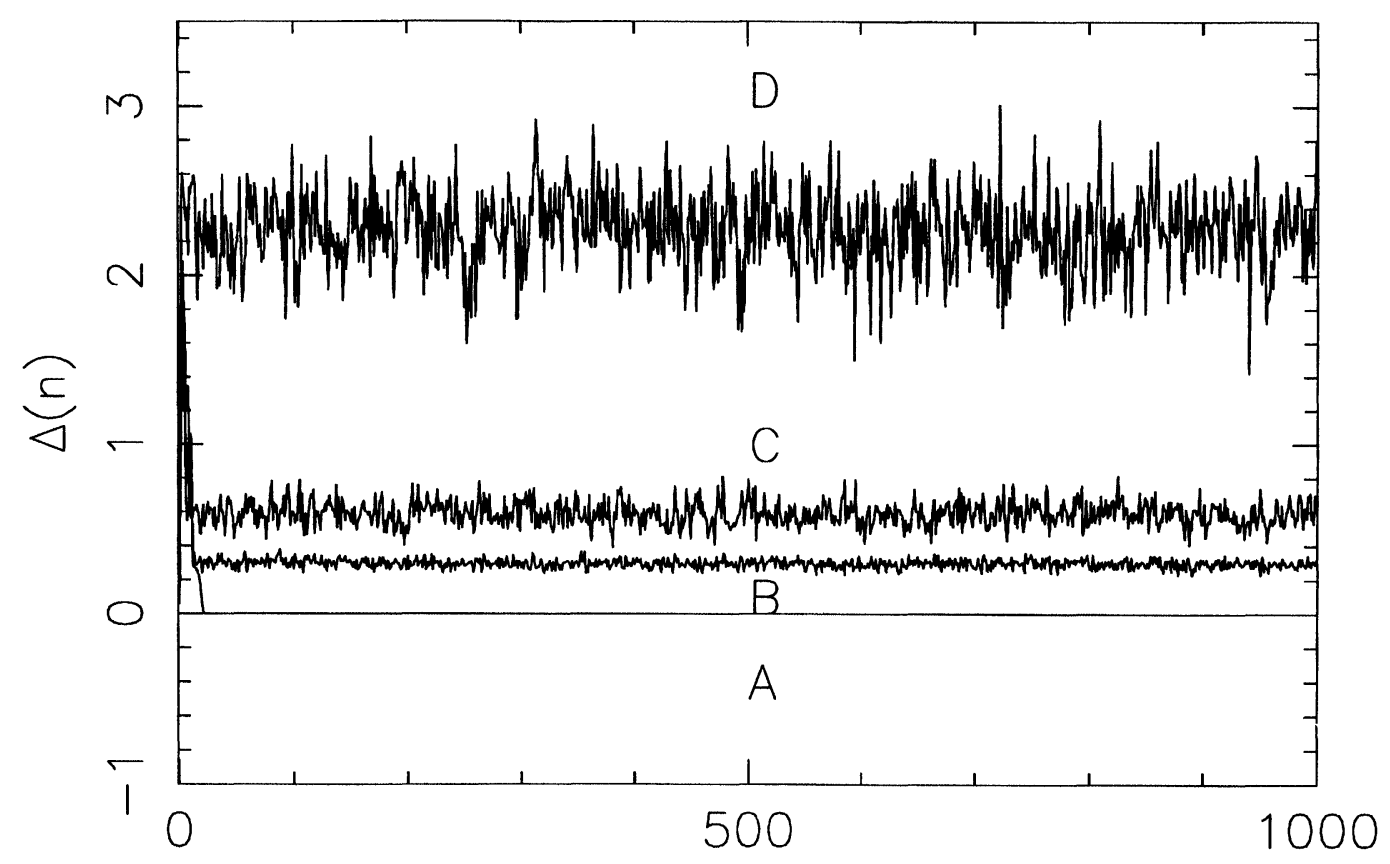

n

FIGURE 3 Synchronization of a CMLRR using Eqs. (3) and (13) in the presence of noise. Here $L=50, \nu=10, \gamma=4, \epsilon=0.1$, $0 \leq \alpha_{i j} \leq \epsilon$ and two feedback functions, fd10 and fd12, corresponding to Eqs. (10) and (12), respectively, are used. In the absence of noise, fd10 and fd12 require 12 and 268 iterations, respectively, for synchronization to $\Delta(n) \leq 1 \times 10^{-10}$. Random numbers $W \eta_{x i}$ and $W \eta_{y i}$ with $-1 \leq \eta_{x i} \leq 1,-1 \leq \eta_{y i} \leq 1$ and $W=0.05$ were added to $X_{n}(i)$ and $Y_{n}(i)$ at every iteration and every site. For this map, values of $\epsilon>0.1$ quickly result in periodic solutions. Those $W \eta_{x i}$ and $W \eta_{y i}$ were used which resulted in bounded solutions of the map. Curve A corresponds to synchronization with common noise $W \eta_{x i}$ or $W \eta_{y i}$ fed to the driver and response systems. Curves B and C, respectively, correspond to synchronization with the faster fd10 and slower fd 12 when different random numbers $W \eta_{x i}$ and $W \eta_{y i}$ are fed to the two systems. Curve $\mathrm{D}$ is generated without any feedback. A comparison of curves $\mathrm{B}$ and $\mathrm{C}$ shows that a better synchronization is obtained by a more efficient feedback function when noise is present. 
presence of weak noise. For simulating noise, we added uniformly distributed random numbers $W \eta_{x i}$ and/or $W \eta_{y i}$ to $X_{n}(i)$ and $Y_{n}(i)$ at every iteration and every site $i$ with $1 \leq i \leq L$. Here $W$ is the fixed strength of the noise while the random numbers are $-1 \leq \eta_{x i} \leq 1$ and $-1 \leq \eta_{y i} \leq 1$. Those $W \eta_{x i}$ and $W \eta_{y i}$ are used which belong to bounded solutions of the map. Figure 3 illustrates the effect of synchronization. When common noise $W \eta_{x i}$ or $W \eta_{y i}$ is fed to the driver and response systems, synchronization is achieved to all figures. However, when the two systems are subjected to different $W \eta_{x i}$ and $W \eta_{y i}$ a degree of synchronization is realized. As expected, the figure shows that the feedback function with a shorter transition time achieve better synchronization than the one with longer transition time. The shortest transition time is 1 . In this ideal case, the dynamical chaos will be synchronized at every iteration but the disagreement between the driver and response systems will remain due to the uncontrolled noise.

In summary, we have used one-dimensional CMLs to simulate spatiotemporal chaos and the method of variable feedback to synchronize this chaos. We have presented a scheme for obtaining feedback functions for model CMLs and have shown that a CML can be synchronized by a multitude of feedback functions with varied efficiencies. We have simulated synchronization in the presence of noise and have found that a degree of synchronization can be achieved with weak noise and fast synchronization schemes. Our numerical results have shown that perfect synchronization can still occur if the driver and response systems are subjected to common noise. We have also shown that sporadic feedback can cause synchronization. It would be very interesting to see how these findings can be put to use in experiments. One area to which our findings may be directly relevant is the storage and retrieval of memory in neural networks.

\section{Acknowledgements}

This research has been supported by an NSERCC grant to M.K. Ali. This work is also supported by funding to Jin-Qing Fang from National Nuclear Industry Science Foundation of China, and China National Project of Science and Technology for Returned Student in Non-Education System. Fang wishes to thank the Department of Physics, the University of Lethbridge for its hospitality.

\section{References}

[1] E. Ott, C. Grebogi and J.A. York, Phys. Rev. Lett., 64, 1196 (1990); W.L. Ditto, S.N. Rauseo and M.L. Spano, Phys. Rev. Lett., 65, 3211 (1990); L. Pecora and T. Carroll, Phys. Rev. Lett., 64, 821 (1990); T. Carroll and L. Pecora, IEEE Trans Circuits and Syst., 38, 453 (1991); E.R. Hunt, Phys. Rev. Lett., 67, 53 (1991); K. Pyragas, Phys. Lett. A, 170, 421 (1992); Z.L. Qu and G. Hu, Phys. Lett. A, 178, 265 (1993); Phys. Rev. Lett., 74, 1736 (1994); L. Kocarev and U. Parlitz, Phys. Rev. Lett., 74, 5028 (1995); J.H. Pen, E.J. Ding, M. Ding and W. Yang, Phys. Rev. Lett., 76, 904 (1996); W.L. Ditto, M.L. Spano and J.F. Lindner, Physica D, 86, 198 (1995); G. Hu, Z. $\mathrm{Qu}$ and $\mathrm{K}$. He, Int. J. Bifur. and Chaos, 5, 901 (1995); Fang Jin-Qing, Progr. in Phys. (in Chinese), 16, 1 (1996) and 16, 137 (1996).

[2] J.A. Sepulchre and A. Babloyantz, Phys. Rev. E, 48, 945 (1994).

[3] Y.C. Lai and C. Grebogi, Phys. Rev. E, 50, 1894 (1994).

[4] D. Auerbach, Phys. Rev. Lett., 72, 1184 (1994).

[5] G. Hu and Z.L. Qu, Phys. Rev. Lett., 72, 68 (1994).

[6] F. Qin, E.E. Wolf and E-.C. Chang, Phys. Rev. Lett., 72, 1459 (1994)

[7] R. Shermer, A. Hubler and N. Packard, Phys. Rev. A, 43, 5642 (1991).

[8] L. Kocarev and U. Parlitz, Phys. Rev. Lett., 77, 2206 (1996).

[9] K. Kaneko, Physica, D, 55, 368 (1992).

[10] T. Shinbrot, Adv. in Phys., 44, 73 (1995).

[11] For the lattice of [10] our feedback function is $G_{n}(i)=$ $\gamma\left[(1-\epsilon) f_{n}(i)+[\kappa /(L-1)] \sum_{j=1, j \neq i}^{L-1}\left(X_{n}(j)-Y_{n}(j)\right)\right]$. 


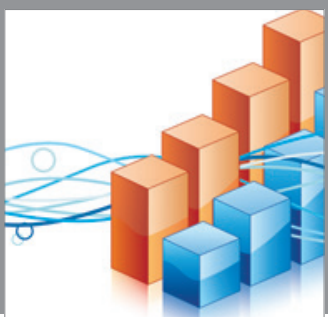

Advances in

Operations Research

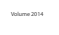

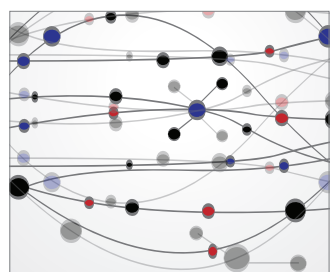

\section{The Scientific} World Journal
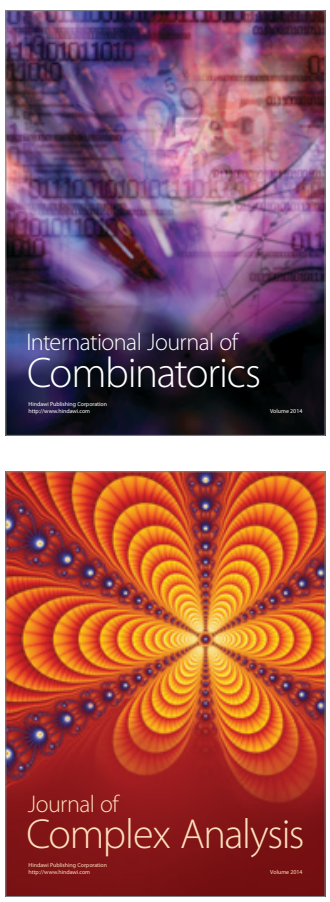

International Journal of

Mathematics and

Mathematical

Sciences
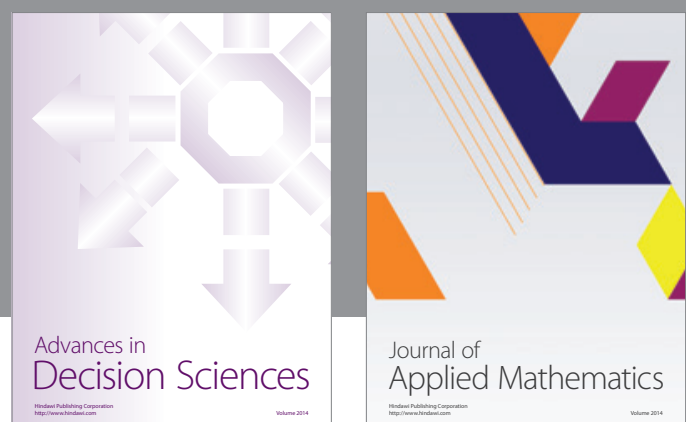

Journal of

Applied Mathematics
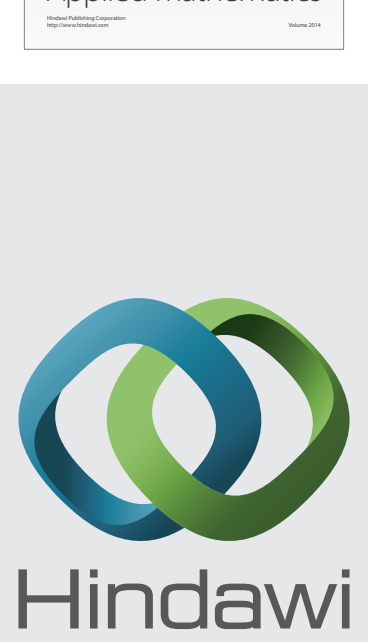

Submit your manuscripts at http://www.hindawi.com
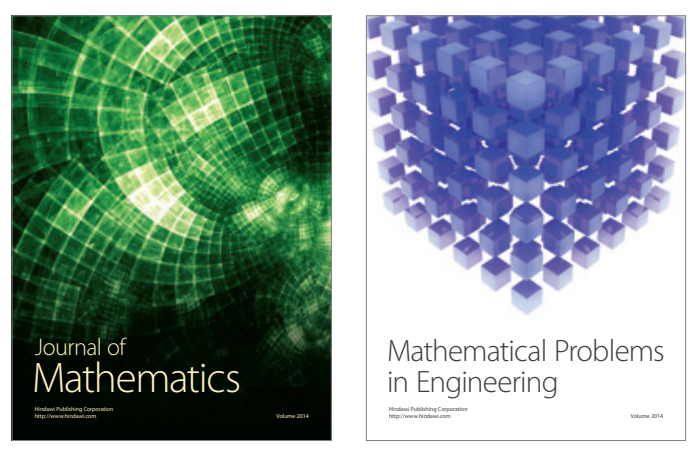

Mathematical Problems in Engineering
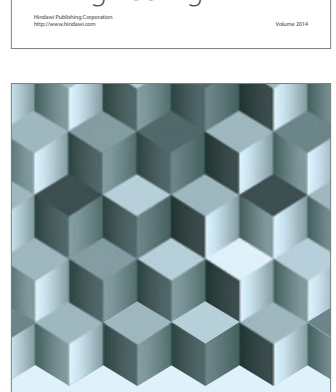

Journal of

Function Spaces
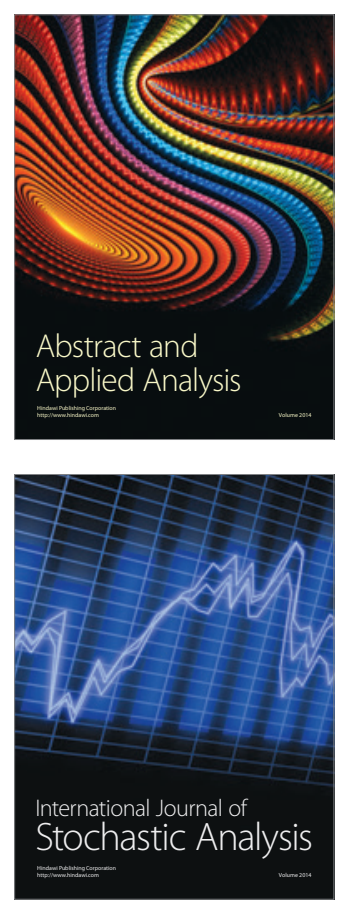

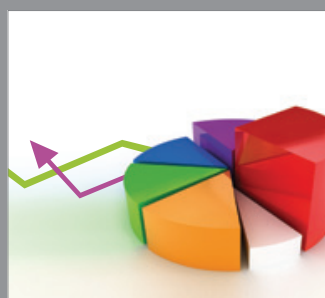

ournal of

Probability and Statistics

Promensencen
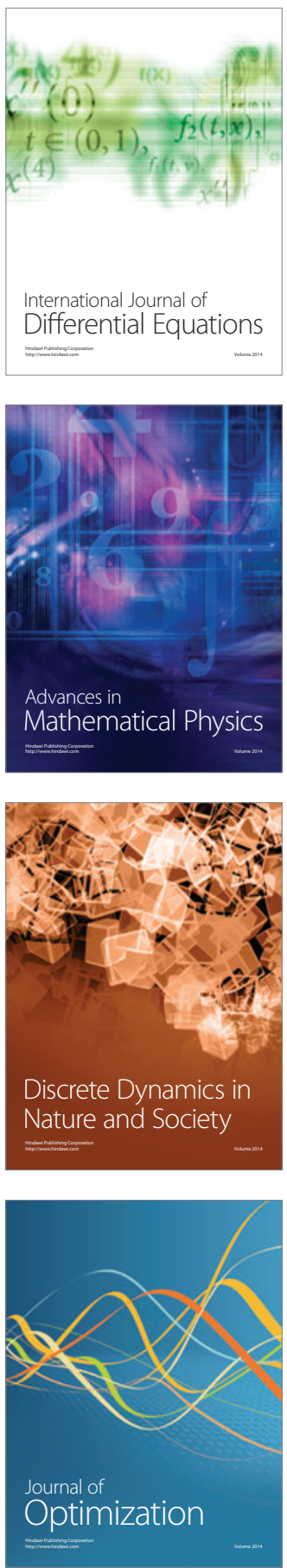